\title{
Estimating organ dose in computed tomography using tube current modulation: A Monte Carlo simulation
}

\author{
V. Hosseinzadeh 1 , H. Ghaffari², A. Rezaeyan ${ }^{2}$, S. Deilami ${ }^{*}$ \\ ${ }^{1}$ Department of Nuclear Engineering, Arsanjan Branch, Islamic Azad University, Arsanjan, Iran \\ ${ }^{2}$ Department of Medical Physics, School of Medicine, Iran University of Medical Sciences, Tehran, Iran \\ ${ }^{3}$ Department of Physics, Arsanjan Branch, Islamic Azad University, Arsanjan, Iran
}

\section{- Original article}

*Corresponding authors: Abolhasan Rezaeyan, Ph.D., E-mail: ahrezaeyan@gmail.com,

Saeid Deilami, Ph.D., E-mail: saeed1542@yahoo.com

Revised: February 2020 Accepted: March 2020

Int. J. Radiat. Res., July 2021; 19(3): $575-581$

DOI: $10.29252 /$ ijrr.19.2.575

\section{ABSTRACT}

Background: The computed tomography (CT) scan delivers a relatively high radiation dose to the patient. One of the critical factors that affects the absorbed dose is the intensity of tube current. The aim of this study is to measure and compare the radiation dose of three radiation-sensitive organs in constant current mode and tube current modulation (TCM) modes. Materials and Methods: CT-scans from the chest and abdomen-pelvis regions of adults in three different current modes were obtained. The absorbed doses of thyroid, lungs, and ovaries were measured using the thermoluminescent dosimeter (TLD) chips embedded in the RANDO phantom. Furthermore, the confirmation of the organ doses was simulated using the Monte Carlo (MC) simulation. The measured doses were evaluated and confirmed by comparison with the simulated doses. Results: The relative differences between the measured and simulated doses for thyroid, lung, and ovary were $-4.7 \%,-1.3 \%$, and $-11.7 \%$ for constant current mode, $-2.2 \%,-11.2 \%$, and $-6.3 \%$ for longitudinal modulation mode, and $0.0 \%,-14.6 \%$, and $-9.9 \%$ for angular modulation mode, respectively. With longitudinal modulation mode, thyroid, lung, and ovary doses were reduced by $34.0 \%, 19.0 \%$, and $19.0 \%$ for the measured doses and $32.0 \%, 26.0 \%$, and $13.0 \%$ for the simulated doses, respectively. The longitudinal modulation mode resulted in a greater dose reduction compared to the angular modulation for both measured and simulated doses. Conclusion: Using TCM resulted in reducing does received by the organs in both measured and simulated doses. The TCM reduces organ dose, which is more evident in the longitudinal modulation.

Keywords: Computed tomography, tube current modulation, organ dose, thermoluminsent dosimeter, Monte Carlo simulation.

\section{INTRODUCTION}

X-ray computed tomography (CT) is known as a main diagnostic tool for a wide range of diseases $(1,2)$. The clinical benefits of CT-scan have shown that CT examinations can deliver relatively high radiation doses to patients, as compared to conventional radiographic examinations, 52 times or even 122 times in some cases. Besides, the number of CT examinations has increased worldwide (3). As a consequence, there are growing concerns regarding the potential cancer risk induced by
CT radiation exposure (3-5). Over the last three decades, the mean radiation dose in the people has dramatically increased, with a nearly sevenfold increment in radiation exposure (6). Taken all together, there are several challenges associated with the justification of CT procedure and dose optimization. To reduce the potential risk of CT-induced cancers, dose optimization is necessary. Multiple parameters and factors affect the patient radiation dose during the CT scan. One of the most crucial factors affecting CT radiation dose received by the patient is X-ray tube current (i.e., current generated in the tube 
due to the flow of electrons inside it). To optimize the dose received by patients, the technologist can adjust the current intensity according to the patient's size and the type of organ being imaged (7). Manufacturers of CT scanners have developed scanners that are capable of modulating the $\mathrm{mA}$ while scanning. At the axial cross-section, the body does not usually have the same thickness in all directions, and the $\mathrm{mA}$ modulation technique focuses on this fact. The rationale behind this technique is that fewer $\mathrm{X}$-ray photons (a smaller $\mathrm{mA}$ ) are required to penetrate thinner tissues and more X-ray photons (a higher $\mathrm{mA}$ ) are needed to penetrate the body's thicker projections $(3,8)$.

Recently, CT scanners are able to modulate tube current as a function of projection angle (angular modulation, XY-plane), longitudinal location along with the patients (z-modulation), or both directions depending on the patient's size and shape as well as the X-ray attenuation characteristics $(9)$. The tube current is modulated as a function of the attenuation level at each $\mathrm{X}$-ray projection view. When the attenuation level at the X-ray projection view is large, the tube current increases to ensure the desired image quality. In contrast, the lower tube current is required when the attenuation level is small. Recent advances in CT technology, especially the implementation of automatic tube current modulation (TCM) techniques, allow for the reduction of radiation exposure (10).

The aim of this study was to measure and compare the radiation dose of three radiation-sensitive organs in the constant current mode and TCM modes including longitudinal and angular modulations. In the present study, we performed experiments to measure and evaluate the doses of three radiation-sensitive organs in the torso region in a constant current mode and current modulation in CT scan protocols of the chest and abdomen-pelvis regions of adults. The novelty of the current study is that our method involves the use of thermoluminescent dosimeter (TLD) chips embedded in the RANDO phantom and confirmation of the organ doses measured by the Monte Carlo (MC) simulation.

\section{MATERIALS AND METHODS}

\section{CT scanner}

This study was conducted using an Activion 16 Multi-slice CT scanner, manufactured by Toshiba Medical Systems Factory (Japan). Toshiba's Activion 16 Multi-slice CT scanner is equipped with a TCM system, allowing the adjustment of the tube current in both angular and longitudinal directions. This scanner acts based on the data obtained from X-ray attenuation in the scanogram (or topogram) images of the patient. The modulation system modulates the tube current to maintain the same standard deviation (SD) that is initially selected by the user during the examination. To ensure the accuracy of the CT scanner performance and validate the results of this study, the latest quality control documents of the device were checked, and the performance of the device was confirmed.

\section{RANDO phantom}

In this study, an anthropomorphic RANDO woman phantom (Radiation Research Center, Shiraz University, Shiraz, Iran) was used. The phantom consists of 35 layers that simulates the anatomical characteristics of a reference female weighing $73.5 \mathrm{~kg}$, an effective atomic number of $7.30 \pm 0.5$, and a density of $1.25 \pm 0.985 \mathrm{~g} / \mathrm{cm}^{3}$. This phantom is made of a real human skeleton consisting of the materials identical to body soft tissues, as well as the substitutes for the lungs, bones, and soft tissues.

\section{Thermoluminescent dosimeters}

In this study, the lithium fluoride TLD (Harshaw chemical company, OH, USA) doped with magnesium and titanium (LiF: $\mathrm{Mg}, \mathrm{Ti}$ (TLD)), known commercially as TLD-100, were used in the form of $3 \times 3 \times 1 \mathrm{~mm}^{3}$ chips. The Harshaw 4500 Manual TLD Reader was applied to readout TLD dosimeters. Before starting the radiation process, to eliminate any previous background irradiation, the dosimeters were placed in an electric oven at $400^{\circ} \mathrm{C}$ for one hour, and then they were returned to the ambient temperature, and again placed in the oven at 100 
${ }^{\circ} \mathrm{C}$ for 2 hours, and kept away from any light and radiation before being used. Five dosimeters were used for calibration and were exposed to $\mathrm{X}$ -rays with high-quality doses similar to the research doses. The results obtained from this experiment are shown in figure 1 . The equation obtained from the diagram was used to calculate the dose in the dosimetry test. Besides, four other dosimeters were used as controls to remove the background rays of the research environment from the obtained doses.

\section{Organ dose measurement}

In the measurement phase, before placing the TLDs in the phantom, a pre-dosimetry test was conducted to accurately determine the scan parameters. Next, the dosimeters were inserted into the phantom and scans from the chest, and abdomen-pelvis were performed in three different modes of tube current (constant current modulation, longitudinal current modulation, and angular current modulation). The current modulation curves and the respective scan parameters for regions of the chest, and abdomen-pelvis CT scans are shown in figure 1 and table 1 . As shown in figure $2 a$ and $b$, the longitudinal TCM curves indicate an approximation of both the angular and longitudinal TCM curves. Also, these curves represent the average tube current values per rotation at each table position in angular and longitudinal TCM CT examinations. There are no image data for the over-ranging region, therefore; the longitudinal TCM curves do not include tube current values in that region.

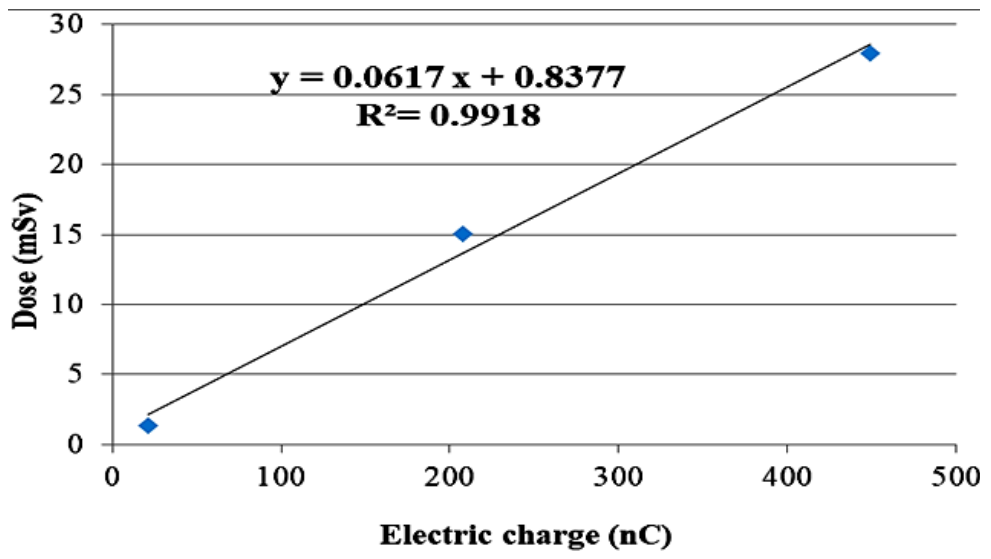

Figure 1. Calibration curve of TLD-100 dosimeters.

Table 1. Scan parameters for adult chest and abdomen-pelvis CT scans using TCM.

\begin{tabular}{|c|c|c|c|c|c|c|}
\hline & \multicolumn{3}{|c|}{ Chest } & \multicolumn{3}{c|}{ Abdomen-pelvis } \\
\cline { 2 - 7 } & constant current & z modulation & xy modulation & constant current & z modulation & xy modulation \\
\hline Scan length (mm) & 300 & 300 & 300 & 440 & 440 & 440 \\
\hline Tube voltage (KV) & 120 & 120 & 120 & 120 & 120 & 120 \\
\hline Tube current (mA) & 40 & $10-40$ & $10-40$ & 110 & $10-110$ & $10-110$ \\
\hline Slice thickness (mm) & 5.0 & 5.0 & 5.0 & 5.0 & 5.0 & 5.0 \\
\hline Rotation time (s) & 1.0 & 1.0 & 1.0 & 1.0 & 1.0 & 1.0 \\
\hline Total scan time (s) & 22.467 & 22.467 & 22.467 & 31.667 & 31.667 & 31.667 \\
\hline Scan-FOV (mm) & $390 \times 300$ & $390 \times 300$ & $390 \times 300$ & $390 \times 440$ & $390 \times 440$ & $390 \times 440$ \\
\hline Target SD & - & 7.5 & 7.5 & - & 7.5 & 7.5 \\
\hline Pitch factor & 15.0 & 15.0 & 15.0 & 15.0 & 15.0 & 15.0 \\
\hline CTDIvol (mGy) & 14.1 & 14.1 & 14.1 & 5.1 & 5.1 & 5.1 \\
\hline DLP (mGy.cm) & 438 & 348.5 & 760.3 & 167.3 & 133.5 & 172.9 \\
\hline
\end{tabular}

The values for CTDIvol and DLP displayed by the scanner. 

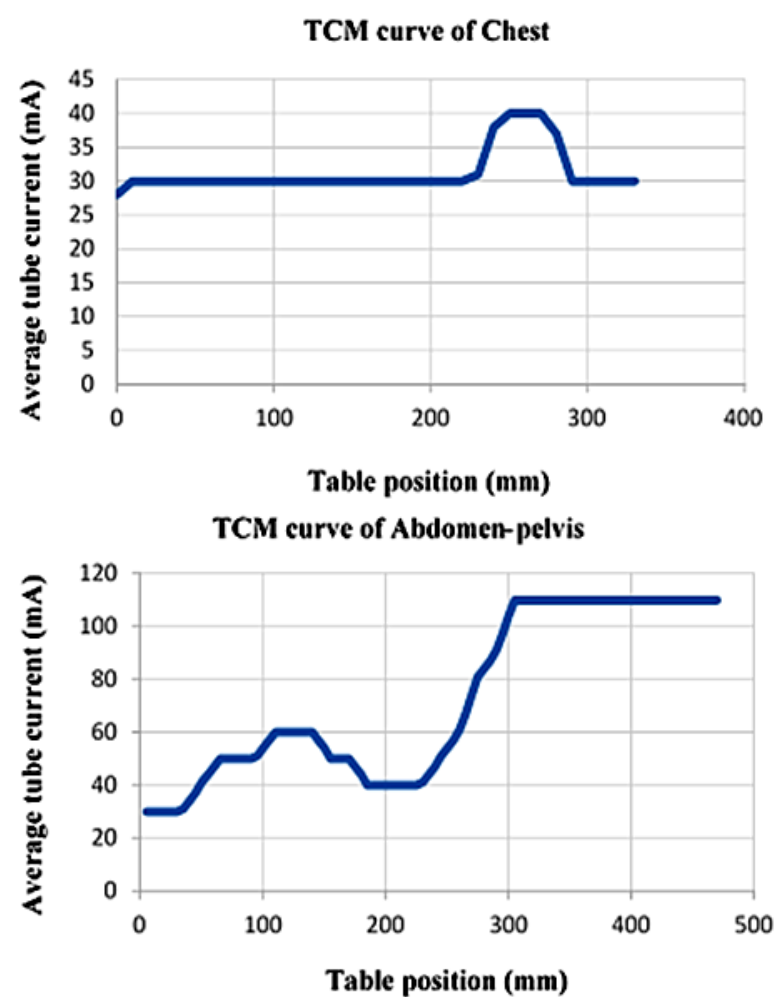

Figure 2. Tube current modulation curve versus table position for (a) chest and (b) abdomen-pelvis CT scans.

Herein, lungs, thyroid (in the chest scan), and ovaries (in the abdomen-pelvis scan) were evaluated as radiation-sensitive organs. A set of prepared TLDs were placed in different points of each organ. The number and location of TLDs in a RANDO view of phantom were as follows; 10 th slice of thyroid slice and number of TLDs 4, lungs 14 and 4 , and ovaries 29 and 5, respectively.

\section{Simulation of organ dose}

The dose of the investigated organs was determined according to the dose distribution of the Monte Carlo (MC) simulation software. The dose simulation with MC requires geometry modeling, x-ray spectrum, and bow-tie-shaped filters of CT scanners. The manufacture provided geometric specifications such as the fan angle and the distance between the focal point and the iso-center of the scanner. To perform the dose simulation using MC, it is necessary to create a Voxelized model of a RANDO phantom based on the CT images.

The X-ray spectrum, the performance of the CT scanner, and all the steps of the dosimetry examination were simulated using the gate code. The dose simulation of the studied organs was conducted using the scan parameters. The scan scope used for the chest and abdomen-pelvis CT included the entire pulmonary region and a region from the diaphragm to the pubic symphysis, respectively. Figure 3 shows CT Images of dose distribution and histograms of dose values in angular modulation for ( $a$ and $b$ ) chest CT scan in slice No. 10 and (c and d) abdomen-pelvis CT scan in slice No. 29. The values of the doses obtained from simulation were compared with the measured doses using the relative differences obtained from the equation 1 :

Difference Rate $(\%)=\frac{\text { simulateddose-measured dose }}{\text { measured dose }} \times 100(1)$ a)

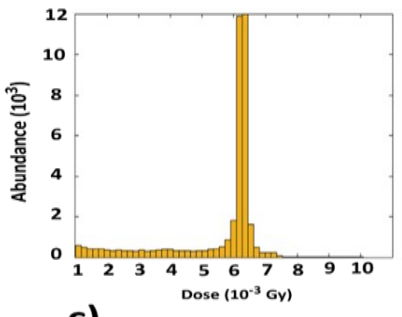

c)

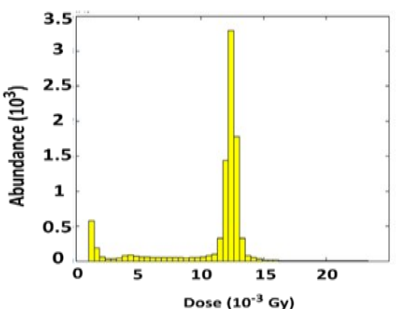

b)

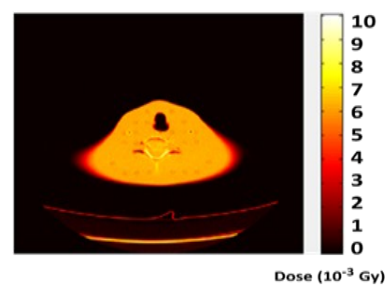

d)

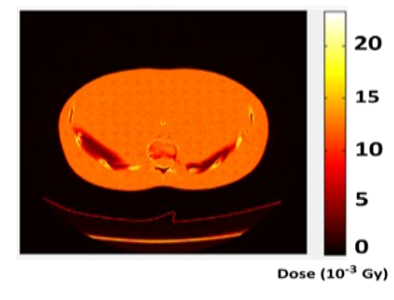

Figure 3. CT Images of dose distribution and histograms of dose values in angular modulation for ( $a$ and $b$ ) chest CT scan in slice No. 10 and ( $c$ and d) abdomen-pelvis CT scan in slice No. 29.

\section{Statistical analysis}

Statistical analysis was performed using the SPSS 22.0 software (SPSS, Chicago, Illinois). The Shapiro-Wilk test was used to investigate the normal distribution of each variable. An independent t-test was used to compare the measured and simulated doses, as well as differences in the doses measured through various tube currents. A $p$-value $<0.05$ was considered statistically significant. 


\section{RESULTS}

Table 2 lists the mean measured and simulated doses for the constant current mode and TCM modes in the thyroid, lung, and ovary. The relative differences between the measured and simulated organ doses for different modes of TCM are outlined in table 2 . The relative differences between the measured and simulated doses for thyroid, lung, and ovary are $-4.7 \%,-1.3 \%$, and $-11.7 \%$ for constant current mode, $-2.2 \%,-11.2 \%$, and $-6.3 \%$ for longitudinal modulation mode, and $0.0 \%,-14.6$ $\%$, and $-9.9 \%$ for angular modulation mode, respectively (table 2 ). No statistically significant difference was found between the measured and simulated doses for any of organs, as observable in table 2.

As observable in table 2, a statistically significant difference in the mean measured doses between the constant current mode and longitudinal modulation mode was found for all organs, as well as for the longitudinal and angular modulation modes. There is a statistically significant difference in the mean measured doses between the constant current mode and angular modulation mode for thyroid and ovary only.

Table 3 outlines the changes in the dose received by the organs after the application of TCM for both measured and simulated doses. With longitudinal modulation mode, thyroid, lung, and ovary doses were reduced by $34.0 \%$, $19.0 \%$, and $19.0 \%$ for the measured doses and $32.0 \%, 26.0 \%$, and $13.0 \%$ for the simulated doses, respectively. As shown in table 3, the longitudinal modulation mode results in a greater dose reduction compared to the angular modulation for both measured and simulated doses. As listed in table 3 , a greater dose reduction for the thyroid compared to the lung and ovary was found using longitudinal modulation mode.

Table 2. Comparison of the measured and simulated doses.

\begin{tabular}{|c|c|c|c|c|c|c|c|c|c|c|c|c|}
\hline \multirow[b]{2}{*}{ Organ } & \multicolumn{3}{|c|}{ Mean measured dose (mGy) } & \multicolumn{3}{|c|}{ Mean simulated dose (mGy) } & \multicolumn{3}{|c|}{ Relative difference (\%) } & \multicolumn{3}{|c|}{$p$-value } \\
\hline & $\begin{array}{c}\text { Constant } \\
\text { current }\end{array}$ & $\begin{array}{l}\text { Longitudinal } \\
\text { modulation }\end{array}$ & $\begin{array}{c}\text { Angular } \\
\text { modulation }\end{array}$ & \begin{tabular}{|c|}
$\begin{array}{c}\text { Constant } \\
\text { current }\end{array}$ \\
\end{tabular} & $\begin{array}{l}\text { Longitudinal } \\
\text { modulation }\end{array}$ & $\begin{array}{c}\text { Angular } \\
\text { modulation }\end{array}$ & $\begin{array}{l}\text { Constant } \\
\text { current }\end{array}$ & $\begin{array}{l}\text { Longitudinal } \\
\text { modulation }\end{array}$ & $\begin{array}{c}\text { Angular } \\
\text { modulation }\end{array}$ & $\begin{array}{c}\text { Constant } \\
\text { current }\end{array}$ & $\begin{array}{l}\text { Longitudinal } \\
\text { modulation }\end{array}$ & $\begin{array}{c}\text { Angular } \\
\text { modulation }\end{array}$ \\
\hline Thyroid & $6.7 \pm 0.5^{a b}$ & $4.4 \pm 0.4^{c}$ & $5.5 \pm 0.5$ & $6.4 \pm 3.5$ & $4.3 \pm 2.9$ & $5.5 \pm 3.3$ & -4.7 & -2.2 & 0 & 0.726 & 0.636 & 0.998 \\
\hline Lung & $7.6 \pm 0.4^{a}$ & $6.2 \pm 0.1^{c}$ & $7.5 \pm 0.3$ & $7.5 \pm 2.4$ & $5.5 \pm 1.6$ & $6.4 \pm 1.3$ & -1.3 & -11.2 & -14.6 & 0.874 & 0.425 & 0.249 \\
\hline Ovary & $\begin{array}{c}13.1 \pm \\
0.4^{a b}\end{array}$ & $10.6 \pm 0.1^{c}$ & $12.1 \pm 0.1$ & $11.5 \pm 1.4$ & $9.9 \pm 1.0$ & $10.9 \pm 1.5$ & -11.7 & -6.3 & -9.9 & 0.371 & 0.561 & 0.533 \\
\hline
\end{tabular}

a: There is a statistically significant difference in the mean measured dose between tube constant current and longitudinal modulation.

b: There is a statistically significant difference in the mean measured dose between tube constant current and angular modulation.

c: There is a statistically significant difference in the mean measured dose between longitudinal and angular modulation.

Table 2. Comparison of the measured and simulated doses.

\begin{tabular}{|c|c|c|c|c|}
\hline \multirow[b]{2}{*}{ Organ } & \multicolumn{2}{|c|}{ \%Dose reduction in measurement } & \multicolumn{2}{|c|}{ \%Dose reduction in simulation } \\
\hline & $\begin{array}{c}\text { constant-longitudinal } \\
\text { modulation }\end{array}$ & $\begin{array}{c}\text { constant-angular } \\
\text { modulation }\end{array}$ & $\begin{array}{c}\text { constant-longitudinal } \\
\text { modulation }\end{array}$ & $\begin{array}{c}\text { constant-angular } \\
\text { modulation }\end{array}$ \\
\hline Thyroid & 34 & 18 & 32 & 14 \\
\hline Lung & 19 & 1.5 & 26 & 14 \\
\hline Ovary & 19 & 8 & 13 & 5 \\
\hline
\end{tabular}

\section{DISCUSSION}

In this study, we measured the radiation dose of three radiation-sensitive organs in the constant current mode and TCM modes (i.e., longitudinal and angular modulation) in adult CT scan protocols of the chest and abdomenpelvis regions. Also, organ doses determined Int. J. Radiat. Res., Vol. 19 No. 3, July 2021 using $\mathrm{MC}$ simulations were validated through comparisons with the doses measured using in-phantom dosimetry with TLD chips. Our results demonstrated that the current modulation resulted in a decrease in the patient dose compared to the constant current. We compared the relative differences between the simulated and measured organ doses for organs 
within the scan ranges for adult chest and abdomen-pelvis CT scans using TCM with the corresponding values for each CT scan using a constant tube current. In the previous study by Fujii etal., (2015) (11) the relative difference between the simulated and measured doses for organs within the scan range were within $3.6 \%$ and $13 \%$ for chest and abdomen-pelvis CT, respectively.

In other study, they reported that the relative difference between the simulated and measured doses for TCM scans using an adult anthropomorphic phantom were within $18.1 \%$ for chest CT and 6.3\% for abdomen-pelvis CT ${ }^{9}$. The dose for each projection angle was measured only in the tube current modulation in chest and abdomen-pelvis CT scans. Evaluation and comparison of the dose values measured from the phantom and the dose determined using MC simulation and the relative differences between them showed that the simulated doses are in good agreement with the measured doses (9). Also, Deak et al. (2008) (12) found relative difference within $10 \%$ between the simulated and measured doses for TCM scans using a liver phantom. As listed in table 2, our results are comparable with above-mentioned studies $(9,11$, 12). Therefore, the simulated doses for CT examinations using TCM agree with the measured doses because the relative differences for TCM examinations are approximately comparable to those for CT examinations when a constant tube current is applied.

The MC simulation has become a common topic for estimating organ absorbed doses $(11,13-$ 15). Huang et al. (2018) (13) assessed the effect of organ-based TCM on reducing lens dose using MC simulation. Fujii et al., (2015) (11) compared the experimental results acquired by the silver-activated phosphor glass dosimeter with the results simulated using ImpactMC (CT Imaging $\mathrm{GmbH}$, Germany) for an adult physical phantom. The percentage difference reported by these studies is about $13 \%$ for organs that were within the scan range. In another investigation conducted by Dabin et al., (2016) (14) organ dose measurements were performed using a five years old anthropomorphic phantom for five different CT scanners from four manufacturers.
The difference between the simulated and measured absorbed doses was about $20 \%$ for most organs, similar to the results found in this study. These results indicated that the major reason for the differences between simulation and measurement approaches are associated with differences in the simulated and measured phantom anatomies.

This study was somewhat limited because most active CT-scan centers are fully booked for emergency and non-emergency patients round-the-clock. Also, with limited number of available TLDs, it was difficult to perform this examination on a large scale. Therefore, this study was performed using only a specific type of scanner and a limited number of organs. This study can be conducted more extensively using CT scanners produced by other manufacturers and with a higher number of slices, as well as on other radiation-sensitive organs. The CT scanner used in this study was not equipped with an organ-based TCM technique; therefore, we suggest that further study can be performed using this technique for dose evaluation.

\section{CONCLUSION}

The thyroid, lung, and ovary are the most radiosensitive organs irradiated during routine thoracic and abdomen-pelvis CT. Hence, we estimated organ dose in routine CT examinations using constant current mode and longitudinal and angular modulation mode. Then, the organ doses determined using MC simulations validated by comparing them with the doses measured using in-phantom dosimetry. Finally, tube current-modulated CT acquisition reduces the radiation dose in these tissues, especially in longitudinal modulation.

\section{Conflicts of interest: Declared none.}

\section{REFERENCES}

1. Brenner DJ and Elliston CD (2004) Estimated radiation risks potentially associated with full-body CT screening. Radiology, 232: 735-8.

Int. J. Radiat. Res., Vol. 19 No. 3, July 2021 
2. Paolicchi F, Bastiani L, Guido D, Dore A, Aringhieri G, Caramella D (2018) Radiation dose exposure in patients affected by lymphoma undergoing repeat $\mathrm{CT}$ examinations: how to manage the radiation dose variability. Radiol Med, 123:191-201.

3. Anam C, Haryanto F, Widita R, Arif I, Dougherty G, McLean D (2018) Volume computed tomography dose index (CTDIvol) and size-specific dose estimate (SSDE) for tube current modulation (TCM) in CT scanning. Int J Radiat Res, 16: 289-97.

4. Zamani H, Falahati F, Omidi R, Abedi-Firouzjah R, Zare MH, Momeni $F$ (2020) Estimating and comparing the radiation cancer risk from cone-beam computed tomography and panoramic radiography in pediatric and adult patients. Int J Radiat Res, 18: 885-93.

5. Chaparian A and Karimi Zarchi H (2018) Assessment of radiation-induced cancer risk to patients undergoing computed tomography angiography scans. Int J Radiat Res, 16: 107-15.

6. Furlow B (2010) Radiation dose in computed tomography. Radiologic Technol, 81: 437-50.

7. Khorramian D, Sistani S, Banaei A, Bijari S (2017) Estimation and assessment of the effective doses for radiosensitive organs in women undergoing chest CT scans with or without automatic exposure control system. Tehran Univ Med J, 75 :496-503.

8. Salimi Y, Deevband MR, Ghafarian P, Ay MR (2018) Uncertainties in effective dose estimation for $\mathrm{CT}$ transmission scan in total body PET-CT imaging with Auto mA3D tube current modulation. Int J Radiat Res, 16: 465-72.
9. Fujii K, Nomura K, Muramatsu Y, Obara S, Akahane K, Kusumoto M (2017) Organ Dose Evaluations Based on Monte Carlo Simulation for CT Examinations Using Tube Current Modulation. Radiat Prot Dosim, 174: 387-94.

10. Graser A, Wintersperger B, Suess C, Reiser MF, Becker CR (2006) Dose reduction and image quality in MDCT colonography using tube current modulation. Am J Roentgenol, 187: 695-701.

11. Fujii K, Nomura K, Muramatsu Y, Takahashi K, Obara S, Akahane K, et al. (2015) Evaluation of organ doses in adult and paediatric CT examinations based on Monte Carlo simulations and in-phantom dosimetry. Radiat Prot Dosim, 165: 166-71.

12. Deak $P$, van Straten $M$, Shrimpton $P C$, Zankl M, Kalender WA (2008) Validation of a Monte Carlo tool for patientspecific dose simulations in multi-slice computed tomography. Eur Radiol, 18: 759-772.

13. Huang Y, Zhuo W, Gao Y, Liu H (2018) Monte Carlo simulation of eye lens dose reduction from $\mathrm{CT}$ scan using organbased tube current modulation. Phys Med, 48: 72-75.

14. Dabin J, Mencarelli A, McMillan D, Romanyukha A, Struelens L, Lee C (2016) Validation of calculation algorithms for organ doses in CT by measurements on a 5 year old paediatric phantom. Phys Med Biol, 61: 4168-4182.

15. Long DJ, Lee C, Tien C, Fisher R, Hoerner MR, Hintenlang D, et al. (2013) Monte Carlo simulations of adult and pediatric computed tomography exams: validation studies of organ doses with physical phantoms. Med Phys, 40: 013901. 
